Service social

\title{
L'émotion et la créativité dans l'étude des processus psychosociaux : une illustration
}

\section{Diane Bernier}

Volume 43, numéro 3, 1994

Intervention individualisée et empowerment

URI : https://id.erudit.org/iderudit/706671ar

DOI : https://doi.org/10.7202/706671ar

Aller au sommaire du numéro

\section{Éditeur(s)}

École de service social de l'Université Laval

ISSN

1708-1734 (numérique)

Découvrir la revue

Citer cet article

Bernier, D. (1994). L'émotion et la créativité dans l'étude des processus psychosociaux : une illustration. Service social, 43(3), 107-121.

https://doi.org/10.7202/706671ar
Résumé de l'article

La réalisation d'une étude sur un processus psychosocial de résolution d'un problème grave (arrêt de travail substantiel pour épuisement professionnel) a inspiré les réflexions méthodologiques de l'auteur. Celles-ci sont pertinentes pour les recherches qualitatives basées sur les témoignages. Les commentaires portent sur le potentiel et la limite de l'émotivité et de la créativité du chercheur. Ils sont regroupés autour de deux pôles : la collecte et l'analyse des données. L'approche non directive permet l'existence d'entrevues dites dramatiques ; l'auteur se sert de l'analogie théâtrale pour définir leurs conditions d'existence. Si l'émotivité du chercheur est sollicitée par ce procédé de collecte des données, sa créativité est stimulée au moment de leur analyse. Des balises sont fournies pour la gestion de la subjectivité du chercheur. 


\section{L'émotion et la créativité dans l'étude des processus psychosociaux: une illustration}

Diane BERNIER

Professeure titulaire

École de service social, Université de Montréal

La réalisation d'une étude du processus de résolution d'une crise d'adaptation (Bernier, 1993)** nous a amenée à réfléchir sur certains aspects méthodologiques de la recherche qualitative. Nos réflexions s'articulent principalement autour du potentiel et de la limite liés à l'émotivité et à la créativité du chercheur.

Dans un premier temps nous situerons nos propos dans le cadre plus général de la recherche sur les processus psychosociaux. Nous ferons par la suite des commentaires plus spécifiques sur la collecte des données et l'analyse du matériel, en présentant quelques avantages et inconvénients que nous avons observés dans notre recherche sur la crise occasionnée par l'épuisement professionnel.

* L'essentiel de ce texte a fait l'objet d'une conférence au congrès de l'ACFAS 1994.

** Cette recherche a été réalisée grâce au financement des Subventions nationales au bien-être social de Développement des ressources humaines Canada. 
Cette illustration apportera, nous semble-t-il, un complément aux propos plus théoriques sur ce sujet formulés par Michel Dorais (1993).

\section{L'ÉTUDE DES PROCESSUS PSYCHOSOCIAUX}

L'intérêt pour un certain nombre de ces processus va de pair avec le développement des disciplines d'intervention. Ainsi des intervenants, parmi lesquels des éducateurs spécialisés, des thérapeutes, des soignants, des animateurs sociaux, se sont intéressés à comprendre les processus de réadaptation sociale (des alcooliques, des toxicomanes, des délinquants, des criminels etc.), les processus de guérison (des troubles psychiques et des maladies physiques, des réactions de stress traumatique, etc.) et les processus de marginalisation (des itinérants, des réfugiés, des sans-emploi, etc.), pour n'en mentionner que quelques-uns. Tous ces processus ont en commun un changement caractérisé par une évolution dans le temps, marqué par une certaine cohérence, réalisé selon une séquence de transformations et aboutissant à un résultat déterminé. Une première exploration de textes portant sur les processus d'intervention (Loyello, 1957; Compton et Gallaway, 1975 ; Zinker, 1978 ; Parad et Parad, 1990 et Bessette, 1994) ne nous ont pas fourni de définition générique du terme processus. Chacun décrit des composantes, un but ou des conditions de réalisations spécifiques. Les quelques définitions suivantes tirées d'un livre de Zinker $(1978: 22,123)$ sur le processus créatif en gestalt illustrent le niveau de définition proposé :

It is in the process of rythmic sharing and active exploration of the client's inner life that his personal structure begins to change.

Therapy is a process of changing awareness and behavior, the sine qua non of the creative process in change: the transformation of one form into another [...].

[...] we modify concrete behavior in a graded and carefully planned manner.

L'auteur s'attarde davantage aux éléments du processus (le changement, le partage et l'exploration) et à son but (la modification de la conscience et du comportement) qu'à sa structure (laquelle est ramenée à une manière de procéder graduée et soigneusement planifiée). La nature et l'ordre de cette procédure ne sont pas précisés, il n'y a pas de référence à un schéma général du déroulement de la 
démarche thérapeutique. Malgré son utilisation fréquente dans le texte, on cherche en vain une définition plus globale du terme processus.

Parmi les composantes des processus psychosociaux, la temporalité mérite une attention particulière. Loyello (1957) souligne "l'importance du temps dans la construction et la reconstruction» de la vie humaine. Ces processus complexes se caractérisent par une certaine durée et, de ce fait, posent des difficultés aux chercheurs. Comment étudier un phénomène qui se déroule sur plusieurs semaines, plusieurs mois, voire plusieurs années? Trois solutions sont possibles: s'en remettre à l'opinion des tiers qui sont des observateurs privilégiés (ex.: Burnett et al., 1994, qui ont consulté des spécialistes pour étudier les aspects normatifs du deuil), suivre le processus à la trace et faire des recherches longitudinales (ex.: Rinfret-Raynor et al., 1991, qui ont évalué l'intervention auprès de femmes violentées) ou les étudier a posteriori en utilisant les perceptions des personnes qui ont vécu ces expériences. Cette dernière option est celle que nous avons retenue pour l'étude de la crise de l'épuisement professionnel. Cette façon d'aborder les processus sert de toile de fond aux réflexions méthodologiques qui constituent l'objet du présent article.

La recherche qualitative se prête particulièrement bien à l'étude des processus basée sur des témoignages. L'intérêt pour l'expérience des sujets qu'elle essaie de systématiser, le traitement de données difficilement quantifiables et l'utilisation d'une méthode d'analyse souple caractérisent la recherche qualitative (Douglas, 1976, dans Deslauriers, 1991). La recherche quantitative, basée sur des données chiffrées, peut aussi être utilisée dans l'étude des processus: elle s'intéresse à leur origine et à leur aboutissement. C'est ainsi qu'elle nous informera de la rémission ou de la morbidité selon diverses typologies de cancer, mais il faut un procédé qualitatif, une description plus approfondie, plus centrée sur le contenu, pour comprendre comment les malades guérissent. L'approche qualitative permet le décodage d'une réalité complexe et changeante: le passage d'une position sociale à une autre, d'un problème à sa solution. Les récits de vie constituent un instrument particulièrement bien adapté à l'étude de ces trajectoires. Ils constituent selon Loicq (1993) une reconstruction de sens, ils décrivent un agencement séquentiel et simultané d'actions ainsi que la durée et l'agencement de phases et de niveaux.

Pour reprendre le schéma proposé par Dorais (1993), la recherche sur laquelle porte notre réflexion constitue une "comparaison de cas ». Elle s'appuie sur l'étude de trente-six récits de vie de deux 
groupes (les intervenants sociaux et les autres professionnels) et porte sur les années qui précèdent et qui suivent l'arrêt de travail lié à l'épuisement professionnel. Une analyse de contenu thématique horizontale a été réalisée en comparant les cas les uns aux autres; elle a été limitée au contenu manifeste. Les catégories ont été établies à l'aide du processus de saturation, c'est-à-dire jusqu'à ce que le matériel $n^{\prime}$ offre plus rien de nouveau en matière d'explicitation d'un concept.

Les théories sur le stress ont servi de cadre conceptuel à cette étude des processus d'ajustement (coping). Selon les témoignages des sujets, la crise du burn-out apparaît comme un événement dévalorisant, marginalisant, qui met parfois en cause la survie professionnelle. Pour comprendre le processus de résolution de cette crise, nous avons cherché à en reconstituer le déroulement à partir de l'arrêt de travail, marqué par les perturbations psychologiques et physiologiques souvent associées à une aversion pour l'activité professionnelle, jusqu'au retour dans l'univers du travail, éventuellement marqué par la stabilité et la satisfaction. La recherche de constantes, d'étapes et de stratégies spécifiquement liées à ce parcours constituait le cœur de cette étude. De celle-ci, nous ne rapporterons que les éléments nécessaires à la compréhension des réflexions méthodologiques qui suivent.

\section{UNE COLLECTE DE DONNÉES PEU BANALE...}

La subjectivité du chercheur qui travaille en recherche qualitative est plus visible. Son analyse n'est pas médiatisée par des tests, qui servent en quelque sorte d'intermédiaires entre lui et le matériel. Cette proximité du matériel revêt des caractéristiques particulières au moment de l'étude des processus d'ajustement à des événements personnels douloureux (crises, institutionnalisations, deuils, situations de violence, etc.). Nous nous attarderons aux instances où la subjectivité du chercheur est particulièrement sollicitée, celles où les entrevues revêtent un caractère dramatique. Notre expérience des récits de vie liés à la crise de l'épuisement professionnel nous a amenée à vivre cette situation. Notre réflexion a été enrichie par la consultation de cinq autres chercheurs engagés dans l'étude d'événements dramatiques. Étant donné la nature très informelle de ces échanges, nous avons choisi de préserver l'anonymat de nos interlocuteurs pour ne pas les lier à l'ensemble de nos propos; à l'occasion, nous nous permettrons de les citer. 


\section{LES ENTREVUES DRAMATIQUES}

Il convient de définir dans un premier temps le terme "dramatique». Les définitions encyclopédiques (Universalis, 1990) et celles de dictionnaires plus anciens (Larousse, 1957) nous renvoient directement au théâtre. Ce n'est que dans un deuxième temps, et dans des éditions plus récentes (Le Petit Larousse, 1993), que le terme drame décrit un événement violent ou tragique, une catastrophe. II nous est apparu pertinent de poursuivre l'analogie théâtrale pour définir les conditions d'existence de ces entrevues dramatiques. Nous en proposons six.

Les trois premières conditions portent sur les procédures qui gouvernent l'existence des entrevues. Dans un premier temps, la rencontre fait l'objet $d^{\prime}$ 'une mise en scène où les rôles sont définis à l'avance: il y aura un narrateur et un confident. Le temps et le lieu de la représentation sont fixés.

Par la suite, l'approche non directive, et plus particulièrement la technique du récit de vie, vient renforcer la dimension théâtrale en permettant à l'émotion de l'interviewé de se développer. Le rythme n'est pas brisé par les questions de l'intervieweur. Le narrateur jouit d'une grande liberté dans la construction de son discours. L'émotion jouit de l'espace nécessaire à son apparition et à son expression. La référence que fait le narrateur à des événements douloureux, de caractère parfois secret, donne lieu à des exposés de grande intensité émotionnelle, possiblement marqués par des pleurs, des difficultés à parler. En raison de ces difficultés, certains chercheurs rapportent même I'annulation de la représentation. Ces aspects rejoignent l'essentiel de la définition des arts théâtraux, si l'on en croit la définition de Bowers (1968:256):

[...] nothing of man originates from deeper and more hidden sources, and nothing surfaces to a more flamboyantly exposed and lasting arena for observation.

De plus, la limite du nombre de rencontres contribue également à l'intensité émotionnelle, à plus forte raison s'il s'agit d'une entrevue unique, comme ce fut notre expérience dans la recherche sur l'épuisement professionnel. Il n'y a pas de temps mort, le narrateur va à l'essentiel pour présenter un condensé des événements. Cette distillation peut avoir été préparée par une réflexion préalable; des thèmes ou des questions lui ayant dans certains cas (dont le nôtre) été soumis avant la représentation. L'essentiel d'un événement significatif est enraciné dans l'émotion. 
Si les trois conditions précédentes se rapportent à la procédure, la suivante concerne le contenu du récit. La narration doit se rapporter à des épisodes pénibles, allant d'une situation de crise à des incidents traumatiques au sens strict du terme. Si I'on se réfère à Schwartz (1991), le terme traumatique devrait être réservé aux événements qui sortent de l'expérience humaine habituelle, qui provoquent une forte réaction de choc ou de stupeur et qui, dans la perception du sujet, menacent sa survie. L'expression "tragique 》 (malgré sa connotation d'abord théâtrale) convient sans doute mieux à une certaine catégorie d'événements aux conséquences catastrophiques. En l'occurrence, la crise associée à l'arrêt de travail pour des motifs d'épuisement professionnel a menacé, pour plus d'un, la survie professionnelle et mérite à ce titre d'être classée parmi cette catégorie d'événements.

Les deux éléments suivants concernent les caractéristiques des acteurs. L'ensemble des conditions précitées sollicitent une écoute particulière de la part du confident; elles permettent, notamment, de porter une attention particulière au non-verbal. « Voir et entendre appartiennent au monde du sensible...», nous rappellent Lae et Murard (1995). De plus, l'intervieweur n'a pas à écrire (le magnétophone enregistre l'entretien) et peut mettre toute sa concentration à écouter. «J'écoutais corps et âme», dira une chercheuse. "C'est facile de vous parler », avouera un interviewé. La nouveauté du sujet pour le chercheur vient sans doute renforcer son intérêt. Enfin, il nous semble probable qu'un chercheur qui s'engage dans ce type de démarche ait des dispositions à l'écoute. Il arrive parfois qu'un chercheur soit très explicite à ce sujet (Lafond, 1993: 29).

[...] nous voulions entendre [...] ce qu'ils avaient à dire. Nous avions une grande motivation à vivre des entrevues intenses où le sujet était dans le cadre le moins rigide possible et où la parole lui était donnée. Nos sentiments d'empathie et de compassion impliquaient ce besoin d'écouter et de laisser le pouvoir aux sujets.

Les narrateurs qui sont émouvants dans leur rôle partagent un certain nombre de caractéristiques. Ils utilisent le langage non verbal, s'expriment dans une langue imagée et colorée; ils ont des qualités de conteurs. Motivés à communiquer, ils sont généralement volontaires pour jouer ce rôle. Cette capacité d'impressionner ne nous semble pas le résultat d'une préméditation ou d'une intention de tromper, mais participe du désir d'être bien compris par le confident, d'éviter les ambiguïtés et les méprises. Ces talents seraient-ils un des biais caractéristiques des sujets qui se prêtent à ce genre d'entretien?

Enfin, un dernier élément nous retient au cœur de I'analogie théâtrale: les interviewés ont conscience d'un auditoire. S'il est vrai, 
selon le point de vue de Goffman (1959), que "l'auditoire» est présent dans la vie quotidienne, à plus forte raison l'est-il dans le cadre d'une représentation théâtrale. Les adultes savent fort bien que les résultats de l'étude seront communiqués à d'autres personnes. "Je suis sortie des entrevues chargée de mission», révélera un des chercheurs consultés. Les narrateurs veulent faire comprendre, veulent aider d'autres personnes, lutter contre l'ignorance et les préjugés. Ces motivations nous ont été clairement explicitées au cours de nos entretiens sur l'épuisement professionnel: «Dites-leur», «Écrivez un livre».

\section{LES SUITES DES ENTREVUES DRAMATIQUES}

La représentation a des effets chez le narrateur et chez le confident. L'expérience a montré que l'entrevue peut être fort dérangeante pour I'interviewé. Il est parfois surpris de ce qu'il a exprimé, de la force de sa propre émotion, bouleversé par ce retour en arrière ou par une prise de conscience nouvelle. Ces réactions ne peuvent être négligées et un soutien thérapeutique doit être mis à la disposition des interviewés. Le chercheur assure les participants qu'un thérapeute est à leur disposition s'ils se sentent troublés à la suite de la rencontre et qu'ils souhaitent parler de leur vécu.

Nous formulons I'hypothèse que l'ampleur de ces réactions varie selon la position du narrateur dans son processus de résolution de la crise ou d'adaptation à un événement tragique; celui qui est plus avancé dans ce processus sera moins perturbé par le rappel occasionné par la mise en scène. Les participants de notre étude sur l'épuisement étaient par définition des "cas à succès", chez qui la crise avait été résolue de façon satisfaisante. Ces réactions extrêmes n'ont pas été apparentes et ne nous ont pas été communiquées à la suite des entrevues.

Par ailleurs, la représentation peut avoir un effet cathartique pour le narrateur. Barrucaud (1970) propose que «la catharsis [...] nécessite la présence combinée de deux processus, la reviviscence affective aussi bien que la mise à distance». Dans le cadre des témoignages, l'expression émotionnelle a un effet libérant. Au cours de nos entrevues sur l'épuisement professionnel, plusieurs sujets nous ont exprimé, et ce malgré le caractère dramatique de l'entrevue, leur intérêt et leur satisfaction de cette occasion de bilan. La présence d'un témoin privilégié contribue au sens de cette synthèse. On sait que le témoignage devant " $\mathrm{d}^{\prime}$ autres" aide les victimes dans leur processus de résolution d'événements traumatiques (Agger et Jensen, 1990 ; Johnson et al., 1995). 
De son côté, le confident ne sort pas toujours indemne de la représentation. L'émotion a été communiquée. À court terme, cette implication émotionnelle risque de le laisser tendu, fatigué, impressionné, voire bouleversé. II aura tendance à y penser beaucoup, à en parler beaucoup (tout en respectant l'anonymat du narrateur). Weinstub (1993), réfléchissant à l'inconfort des professionnels devant la souffrance de leurs clients, reconnaît les lacunes du droit et de la médecine dans le traitement des victimes de tragédie. Les chercheurs échapperaient-ils à cet inconfort?

La réaction émotionnelle du confident constitue à la fois une ressource et un handicap. C'est un atout précieux pour comprendre et, selon l'expression d'un chercheur consulté, pour "construire l'objet de recherche». L'émotion sous-jacente (ex. : la honte, la peine, I'impuissance) véhiculée par le narrateur a été ressentie par le confident, qui arrive à la décoder et à la nommer. Pour y arriver, sa subjectivité a été poussée jusqu'à une position limite.

Cet atout pour la compréhension peut devenir un handicap pour I'analyse. Si la communication émotionnelle a aidé à comprendre de I'intérieur, c'est qu'elle a, dans une certaine mesure, amené le confident à épouser momentanément le point de vue du narrateur. De la même façon qu'au théâtre, le spectateur est amené à vibrer à l'unisson avec les acteurs. Plus la démonstration est convaincante, plus il sera difficile au chercheur de s'en détacher pour adopter un autre point de vue. Et pourtant, I'analyse exige un regard neuf. Comment concilier cette exigence d'extériorité avec la compréhension intime qu'il a acquise du point de vue du narrateur? La sensibilité du chercheur ne s'éteint pas avec les feux de la rampe. Son émotion subsiste un certain temps... Une chercheuse avoue: "Je suis restée avec les phrases dans la tête»; elle a mis un an à prendre le recul nécessaire pour compléter son analyse. Un premier travail de confrontation avec le matériel facilite cette distanciation et permet, dans un deuxième temps, des élaborations plus originales. Le chercheur qui n'a pas repris un minimum de neutralité se trahit habituellement dans le langage qu'il utilise pour rapporter ses observations. Nous en avons fait l'expérience lorsque nous avons soumis le premier manuscrit de notre rapport de recherche portant sur des entrevues dramatiques: un des lecteurs a souligné quelques passages où le langage utilisé manquait de neutralité. Nous avons par la suite apporté les corrections nécessaires. Le passage du temps ne suffit pas au chercheur pour gérer ses émotions, d'autres modalités sont nécessaires; elles sont décrites en conclusion. 


\section{UNE ANALYSE CRÉATRICE...}

Si l'émotivité du chercheur a été fortement sollicitée dans la collecte des données, sa créativité l'est tout autant à d'autres étapes.

\section{L'absence de catégories préalables}

Parmi les procédures de l'analyse qualitative, celle qui autorise la création a posteriori des catégories présente beaucoup d'intérêt dans l'étude des processus peu connus. Elle permet une disponibilité totale vis-à-vis des données recueillies. Le but n'est pas d'assigner le matériel à des cases préétablies, mais plutôt de créer ces cases. C'est la situation dans laquelle nous nous trouvions au début de notre recherche sur l'épuisement professionnel.

On a établi l'existence d'étapes dans certains processus; les phases du deuil de Kübler-Ross (1969) sont sans doute parmi les plus connues. Existe-t-il des étapes dans le processus de résolution d'une crise associée à l'arrêt de travail causé par une l'épuisement? L'évolution de l'épuisement professionnel grave par suite de l'incapacité de travailler a été mentionnée dans quelques textes à contenu plus clinique avec des exemples ponctuels à I'appui (Aubert et Pagès, 1989 ; Freudenberger, 1986; Maslach, 1982 ; Pines et al.,1982 ), mais elle $\mathrm{n}$ 'a jamais fait l'objet d'une documentation systématique. De ce point de vue, la question était sans réponse.

Notre expérience clinique et notre connaissance des écrits sur le stress nous permettaient d'envisager l'existence de stratégies d'ordre cognitif (ex.: changer sa façon de voir les choses) et comportemental (ex.: apprendre à mettre des limites); c'est ce que nous nous attendions de retrouver. L'émergence d'un parcours marqué par des étapes facilement identifiables a constitué une révélation pour nous, alors que l'absence d'une catégorisation préalable nous a permis de rester près du matériel et d'établir des rubriques pertinentes. Notons que la nomenclature des étapes est basée sur la description faite par les participants des activités qui caractérisent chacune d'entre elles. La reconnaissance du problème, la distanciation des sources de stress, la restauration des capacités, la remise en question des valeurs, l'exploration des possibles et la rupture ont été définies comme les six étapes générales du processus de résolution de la crise associée à l'épuisement professionnel.

Ce qui nous semble maintenant une quasi-évidence est le résultat d'un point de vue extérieur et d'une vision d'ensemble. Lorsqu'on les a interrogés, à la fin de l'entrevue, sur leur perception d'étapes vécues 
dans leur processus de résolution, les participants sont très rarement arrivés à déterminer des séquences et à mettre des titres sur leur vécu; personne n'est arrivé à décrire comme tel l'ensemble des étapes que nous avons observées. Cette première catégorisation d'une séquence de phases est le résultat de l'analyse du chercheur.

Dans un premier temps, nous avons été déçue par le caractère très concret des étapes identifiées. Nous avions espéré une plus grande élaboration des mécanismes psychiques en cause dans le processus, mais ce genre de perception était peu accessible aux interviewés. Ainsi une participante, par ailleurs bien articulée, n'a pas pu nous expliquer pourquoi ou comment sa tristesse diminuait avec le temps. Nous nous sommes donc limitée à une définition plus "extérieure » des six étapes. Par ailleurs, le niveau de formulations explicitées rend bien compte de la portée psychosociale de ce processus. Mis à part la restauration des capacités, toutes les autres étapes ont un lien avec I'environnement.

La créativité du chercheur n'est pas sans frontières; ainsi, des auteurs ont formulé des balises pour la création de catégories. Nous nous sommes référée à celles de Lazarsfeld (1972: 227): I'articulation, la correction logique, I'adaptation à la structure de la situation globale et I'adaptation au cadre de référence des répondants ${ }^{1}$. La dimension sociale de nos catégories satisfait particulièrement bien les troisième et quatrième critères: ces catégories sont bien adaptées au processus à l'étude, rendent compte d'un ensemble d'éléments et restent près du niveau d'explication fourni par les sujets.

\section{La souplesse de l'analyse}

La procédure d'analyse qualitative n'étant pas figée dans une formule, une équation ou un test, elle permet une réorganisation du matériel lorsqu'une première catégorisation n'est pas pertinente. Ainsi, notre catégorisation initiale comportait huit étapes : la reconnaissance du problème, la distanciation du travail, la restauration des capacités, la remise en question des valeurs, la reprise de contact avec l'univers du travail, les démarches de planification en vue d'un travail satisfaisant, le retour au milieu de travail et le départ de la niche initiale. L'étude des quatre premières s'est déroulée sans encombre, mais I'organisation des quatre dernières étapes n'était pas satisfaisante, leur

1. Traduction libre de l'auteure. 
contenu n'était pas mutuellement exclusif et la nomenclature utilisée ne permettait pas de regrouper toutes les expériences. Après réflexion et relectures, nous avons fusionné les cinquième, sixième et septième catégories et trouvé une appellation à portée plus large : I'exploration des possibilités de travail. Par suite de l'analyse de la huitième étape, nous en avons modifié le titre (rupture d'avec les sources de stress), afin de mieux rendre compte du fil conducteur commun à tous les récits. Ces modifications, faites en cours de route, contribuent à la pertinence et à l'enrichissement de l'analyse. Cette réorganisation successive des données a d'ailleurs été décrite par Glazer et Strauss (1967) dans leur élaboration des stratégies d'analyse qualitative. De leur point de vue, la comparaison des incidents "facilite la génération de théories sur les processus, les séquences et le changement relatif aux organisations, aux positions et à l'interaction sociale ${ }^{2} »$.

L'interaction entre la lecture et l'analyse est recommandée dans I'analyse de contenu qualitative (Deslauriers, 1991). Nous en avons expérimenté l'utilité au cours de cette recherche sur le processus de résolution de la crise. Après l'analyse des étapes, il restait plus du tiers du matériel qui apparaissait périphérique par rapport au sujet. Nous projetions de le présenter tantôt en toile de fond, tantôt en conclusion; nous l'avons déplacé, renommé, replacé, mais aucune solution ne nous semblait satisfaisante... C'est en cherchant autre chose (une meilleure définition du concept de coping) que nous avons trouvé le concept organisateur de cette grande partie du matériel. Un texte de Schlenker (1987) propose que des mécanismes particuliers entrent en jeu lorsque les images identitaires sont menacées: la personne tente de se rassurer, de comprendre et de se renforcer. Si cette trouvaille au sujet des stratégies identitaires donnait un sens, entre autres, aux longues narrations sur les causes de la crise, ce dont nous étions fort satisfaite, elle nous a par ailleurs forcée, à un moment où l'analyse était terminée, à retourner au matériel de base pour documenter l'atteinte à l'identité et les façons de se rassurer quant à cette menace. L'utilisation de ces stratégies n'étant pas liée à des moments précis, elles n'avaient donc pas trouvé place dans la catégorisation de matériel décrivant le contenu et la chronologie des étapes. Dans l'expérience de recherche dont nous témoignons ici, la souplesse de l'analyse qualitative nous a permis un aller-retour du matériel aux écrits particulièrement fructueux. Cette procédure permet d'alimenter la créativité du chercheur.

2. Traduction libre de l'auteure. 


\section{CONCLUSION}

Notre réflexion sur les avantages et les inconvénients de la recherche qualitative a été orientée par nos travaux personnels dans l'étude des processus. Nous n'avons pas la prétention d'avoir fait une présentation exhaustive de ces aspects. Par ailleurs, nous croyons opportun d'approfondir, en conclusion, la façon de maximiser les avantages et de minimiser les inconvénients déjà soulignés.

L'absence de contraintes préétablies sur le plan conceptuel laisse beaucoup de place à la créativité du chercheur. Ainsi, la souplesse de l'analyse met à contribution son jugement personnel et son esprit d'initiative. Ces aptitudes, inhérentes à ce type de recherche, sont à valoriser chez le jeune chercheur qui peut manquer d'assurance dans cette procédure moins balisée. Le feed-back des collègues constitue un soutien précieux dans les moments de doute ou de confusion relative. Les lecteurs de manuscrits jouent à cet égard un rôle important. Nous avons beaucoup bénéficié de commentaires de nos lecteurs "profanes » et "professionnels » (ces termes se référant à leur connaissance tantôt du sujet, tantôt de la méthodologie). La souplesse de la recherche qualitative permet de mettre leurs suggestions à profit dans l'organisation et la présentation du matériel.

Nous avons développé dans ce texte la dimension émotionnelle des entrevues non directives dans l'étude des processus, dimension mentionnée ailleurs (Veyne, 1971: 21-22, Leclerc-Olive, 1993) comme le ... «pathos qui accompagne la plupart des récits autobiographiques... » ou plus sobrement comme «le poids socio-affectif de l'entretien biographique» (Desmarais, 1986). La gestion de l'émotion passe à notre avis par son expression, ce qui en minimise l'effet submergeant; la nier serait dangereux. Cette expression peut revêtir plusieurs formes, verbales ou écrites. Le journal de bord du chercheur peut à l'occasion servir d'exutoire au trop-plein émotionnel. Le travail à deux ou à plusieurs chercheurs constitue un atout de ce point de vue.

Au moment de I'analyse, les lectures servent également de contrepoids à l'impact émotionnel des entrevues dramatiques. Elles présentent une vision plus sobre, parfois divergente qui remet en question le point de vue présenté de façon si impressionnante par les interviewés. À titre d'exemple, la position de victime révélée par quelques-uns de nos sujets ne résiste pas à une analyse plus objective du vécu professionnel en milieu de travail.

Compte tenu des impératifs de la production rapide des travaux de recherche, il est probablement utopique de préconiser une analyse 
où la rédaction finale se réaliserait dans un deuxième temps, après une période de "décantation émotionnelle». Cependant, les articles subséquents à l'écriture du rapport de recherche sont l'occasion de reformulations, d'ajouts ou de synthèse. Le chercheur solitaire a intérêt à se prévaloir de ces mécanismes.

Enfin, les procédures de validation propres à la recherche qualitative viennent rassurer le chercheur quant à l'objectivité de ses analyses. En effet, ses conclusions peuvent être soumises aux participants soit dans un contexte de groupe ou de façon individuelle. Nous avons soumis notre manuscrit à tous les interviewés pour solliciter leur approbation des extraits qui les concernaient. Ils ont eu à cette occasion le loisir de nous transmettre leurs commentaires sur le contenu. Un seul participant nous a reflété sa distance vis-à-vis du processus décrit. L'expérience des personnes qui connaissent bien le phénomène peut aussi être mise à profit dans le processus de validation. Nous avons utilisé des thérapeutes comme lecteurs de la version initiale.

Ces derniers nous ont confirmé la similitude du processus décrit avec leur expérience clinique.

Notre expérience nous amène à conclure que l'intérêt et la fécondité de la recherche basée sur les témoignages dépassent ses écueils. En utilisant les mécanismes appropriés, le chercheur, même solitaire, peut exécuter un travail de grande qualité. 


\section{Références bibliographiques}

AGGER, I. et S.B. JENSEN (1990). "Testimony as ritual and evidence in psychotherapy for political refugees », Journal of Traumatic Stress, vol. 3, n $1: 115-130$.

AuBert, N. et M. PAGÈs (1989). Le stress professionnel. Paris : Klincksieck.

BARRUCAUD, D. (1970). La catharsis dans le théâtre, la psychanalyse et la psychothérapie de groupe. Paris : Epi.

BERNIER, D. (1993). La crise du burnout. S'en remettre, c'est refaire sa vie. Montréal: Stanké.

BESSETTE, L. (dir.) (1994). Le processus de guérison : par-delà la souffrance ou la mort. Québec: MNH.

BOWERS, F. (1968). International Encyclopedia of the Social Sciences, vol. 4. David L. Sields (dir.). The Mcmillan Co. and the Free Press.

Burnett, P., W. Middleton, B. Raphael, M. Dunne, A. Moylan et N. MARTINEK (1994). "Concept of normal bereavement», Journal of Traumatic Stress, vol. 7, n ${ }^{\circ} 1: 123-128$.

Compton, B.R. et B. Gallaway (1975). Social Work Processes. Illinois: Dorsey Press.

DESLAURIERS, J.P. (1991). Recherche qualitative: guide pratique. Montréal: McGraw-Hill.

Desmarais, D. et P. Grell (1986). Les récits de vie: théorie, méthode et trajectoires types. Montréal: Éditions Saint-Martin.

DORAIS, M. (1993). "Diversité et créativité en recherche qualitative», Service social, vol. 42, $\mathrm{n}^{\circ} 2: 7-27$.

Encyclopaedia Universalis, corpus 7 (1990). Paris.

FREUDENBERGER, H.G. (1982). L'épuisement professionnel : la "brûlure interne ». Ottawa: Iredi.

GLAZER, B.G. et A.L. StRAuSS (1967). The discovery of grounded theory: strategies for qualitative research. Chicago: Aldine Publishing Company.

GOFFMAN, E. (1959). The presentation of self in everyday life. New York: Doubleday Anchor.

JOHNSON, D.R., S.C. FELdMAN, H. LUBIN et S.M. SOUTHWICK (1995). "The therapeutic use of ritual and ceremony in the treatment of post-traumatic stress disorder», Journal of Traumatic Stress, vol. 8, $\mathrm{n}^{\circ} 2$ : 283-298.

KÜBLER-ROSS, E. (1969). On death and dying. New York: McMillan.

LAE, J.F. et N. MURARD (1995). Les récits du malheur. Paris: Descartes et Cie.

LAFOND, C. (1993). Avoir une mère atteinte de maladie mentale: trois histoires de cas. Mémoire de maîtrise, École de service social, Université de Montréal.

Larousse (1957). Paris: Larousse.

Petit Larousse illustré (1993). Paris: Larousse.

LAZARSFELD, P.F. (1972). Qualitative Analysis: Historical and critical essays. Boston: Allyn and Bacon, Inc. 
LAUDERDALE, M. (1982). Burnout, strategies for personal and organizational life: Speculations on evolving paradigms. Austin : Learning Concepts, University Associates.

LECLERC-OlIVE, M. (1993). "Les événements biographiques», dans Vincent de Gaulejac et Shirley Roy (dir.), Sociologies cliniques. Marseille: Hommes et Perspectives, Epi.

LOICQ, F. (1993). "Se raconter sous le regard de I'institution», dans Vincent de Gaulejac et Shirley Roy (dir.), Sociologies cliniques. Marseille: Hommes et Perspectives, Epi.

LOYELLO, W. (1957) . "L'intégration de la temporalité et le processus thérapeutique», dans Le processus psychothérapeutique: formes et évaluations. Annales de psychothérapie, publiées par la Société de recherches psychothérapiques de langue française. Paris: Éditions ESF.

MASLACH, C. (1982). Burn-out : the cost of caring. Englewood Cliffs, NJ: Prentice- Hall.

PARAD, H.J. et L.G. PARAD (1990). Crisis intervention. Book 2 : The practitioner's sourcebook for brief therapy. Wisconsin: Family Service America.

PINES, A. et E. ARONSON (1988). Career burnout: causes and cures. New York: The Free Press.

Rinfret-Raynor, M., A. Paquet-Deehy, G. Larouche et S. CANTin (1991). Intervenir auprès des femmes violentées : évaluation de l'efficacité d'un modèle féministe. Résumé d'un rapport de recherche, Université de Montréal.

SCHLENKER, B.R. (1987). "Threats to identity : self-identification and social stress », dans C.R. Snyder et C. E. Ford (dir.), Coping with negative life events. New York and London : Plenum Press.

SCHWARTZ, L.S. (1990). "A biopsychosocial treatment approach to post-traumatic disorders", Journal or Traumatic Stress, vol. 3, $\mathrm{n}^{\circ} 2$ : 221-238.

Weinstub, D. (1994). "Problems of treating tragedy: The limits of law and medecine», dans Luc Bessette (dir.), Le processus de guérison: par-delà la souffrance ou la mort. Québec: MNH.

ZINKER, J. (1978). Creative process in gestalt therapy. New York: Vintage Books. 